TRANSACTIONS OF THE

AMERICAN MATHEMATICAL SOCIETY

Volume 358, Number 8 , Pages 3733-3749

S 0002-9947(05)03875-4

Article electronically published on December 27, 2005

\title{
ON HIGHER SYZYGIES OF RULED SURFACES
}

\author{
EUISUNG PARK
}

\begin{abstract}
We study higher syzygies of a ruled surface $X$ over a curve of genus $g$ with the numerical invariant $e$. Let $L \in \operatorname{Pic} X$ be a line bundle in the numerical class of $a C_{0}+b f$. We prove that for $0 \leq e \leq g-3, L$ satisfies property $N_{p}$ if $a \geq p+2$ and $b-a e \geq 3 g-1-e+p$, and for $e \geq g-2, L$ satisfies property $N_{p}$ if $a \geq p+2$ and $b-a e \geq 2 g+1+p$. By using these facts, we obtain Mukai-type results. For ample line bundles $A_{i}$, we show that $K_{X}+A_{1}+\cdots+A_{q}$ satisfies property $N_{p}$ when $0 \leq e<\frac{g-3}{2}$ and $q \geq g-2 e+1+p$ or when $e \geq \frac{g-3}{2}$ and $q \geq p+4$. Therefore we prove Mukai's conjecture for ruled surface with $e \geq \frac{g-3}{2}$. We also prove that when $X$ is an elliptic ruled surface with $e \geq 0$, $L$ satisfies property $N_{p}$ if and only if $a \geq 1$ and $b-a e \geq 3+p$.
\end{abstract}

\section{INTRODUCTION}

In this article we study higher syzygies of irrational ruled surfaces. Let $X$ be a smooth projective variety and let $L \in \operatorname{Pic} X$ be a very ample line bundle. Consider the embedding

$$
X \hookrightarrow \mathbb{P} H^{0}(X, L)=\mathbb{P}
$$

defined by the complete linear system of $L$. Let $S$ be the homogeneous coordinate ring of $\mathbb{P}$ and consider the graded $S$-module $E=\bigoplus_{n \in \mathbb{Z}} H^{0}\left(X, L^{n}\right)$. Let

$$
\cdots \rightarrow \bigoplus_{j} S^{k_{i, j}}(-i-j) \rightarrow \cdots \rightarrow \bigoplus_{j} S^{k_{1, j}}(-1-j) \rightarrow \bigoplus_{j} S^{k_{0, j}}(-j) \rightarrow E \rightarrow 0
$$

be a minimal graded free resolution of $E$. A main goal to study higher syzygies is to interpret the information carried by the graded Betti number $k_{i, j}$. In particular, the distribution of zeroes in the Betti table enables us to understand the geometry of $X \hookrightarrow \mathbb{P}$. Along this point of view, many people have studied the so-called property $N_{p}$ which means that the first few modules of syzygies are as simple as possible.

Definition 1.1 (Green-Lazarsfeld, [9]). $(X, L)$ satisfies property $N_{p}$ if $k_{i, j}=0$ for $0 \leq i \leq p$ and $j \geq 2$. Equivalently, property $N_{p}$ holds for $X \hookrightarrow \mathbb{P}$ if $E$ admits a minimal free resolution of the form

$$
\cdots \rightarrow S^{m_{p}}(-p-1) \rightarrow \cdots \rightarrow S^{m_{2}}(-3) \rightarrow S^{m_{1}}(-2) \rightarrow S \rightarrow E \rightarrow 0 .
$$

Therefore property $N_{0}$ holds if and only if $X \hookrightarrow \mathbb{P} H^{0}(X, L)$ is a projectively normal embedding, property $N_{1}$ holds if and only if property $N_{0}$ is satisfied and the homogeneous ideal is generated by quadrics, and property $N_{p}$ holds for $p \geq 2$

Received by the editors January 26, 2004 and, in revised form, October 16, 2004.

2000 Mathematics Subject Classification. Primary 13D02, 14J26, 14 N05.

The author was supported by Korea Research Foundation Grant (KRF-2002-070-C00003). 
if and only if it has property $N_{0}$ and $N_{1}$ and the $k^{\text {th }}$ syzygies among the quadrics are generated by linear syzygies for all $1 \leq k \leq p-1$.

Concerning higher syzygies, M. Green 8 obtained the first general result. He proved that if $C$ is a smooth curve of genus $g$ and if $\operatorname{deg}(L) \geq 2 g+1+p$, then $(C, L)$ satisfies property $N_{p}$. This result was rediscovered by M. Green and R. Lazarsfeld [10. Also they classified all pairs $(C, L)$ for which the " $2 g+1+p$ " theorem is optimal.

Theorem 1.1 (Green-Lazarsfeld, [10]). Let $L$ be a line bundle of degree $2 g+$ $p(p \geq 1)$ on a smooth projective curve $X$ of genus $g$, defining an embedding $C \hookrightarrow$ $\mathbb{P} H^{0}(C, L)=\mathbb{P}^{g+p}$. Then $(C, L)$ fails to satisfy property $N_{p}$ if and only if either

(i) $C$ is hyperelliptic or

(ii) $C \hookrightarrow \mathbb{P}^{g+p}$ has a $(p+2)$-secant $p$-plane, i.e., $H^{0}\left(C, L-K_{C}\right) \neq 0$ or equivalently $L=K_{C}+D$ for some effective divisor $D$ of degree $p+2$.

Then Mukai observed that Green's Theorem implies for ample line bundle $A$ on a smooth curve $C, K_{C}+(p+3) A$ satisfies property $N_{p}$. Also, he has conjectured that

( ) Mukai's Conjecture. For a smooth projective surface $S$ and an ample line bundle $A \in \operatorname{Pic} S, K_{S}+(4+p) A$ satisfies property $N_{p}$.

Though this conjecture is still open, some progress has been made by D. Butler for ruled surfaces [1] and by F. J. Gallego and B. P. Purnaprajna for elliptic ruled surfaces [3, 4, surfaces of nonnegative Kodaira dimension [5, 6] and rational surfaces [7.

The aim of this article is to study higher syzygies of ruled surfaces over an irrational curve with numerical invariant $e \geq 0$. More precisely, we refine results in [1] and [4]. We will follow the notation and terminology of R. Hartshorne's book [11, $\mathrm{V} \S 2$. Let $C$ be a smooth projective curve of genus $g$, and let $\mathcal{E}$ be a vector bundle of rank 2 on $C$ which is normalized, i.e., $H^{0}(C, \mathcal{E}) \neq 0$, while $H^{0}\left(C, \mathcal{E} \otimes \mathcal{O}_{C}(D)\right)=0$ for every divisor $D$ of negative degree. We set

$$
\mathfrak{e}=\bigwedge^{2} \mathcal{E} \text { and } e=-\operatorname{deg}(\mathfrak{e})
$$

Let $X=\mathbb{P}_{C}(\mathcal{E})$ be the associated ruled surface with projection morphism $\pi: X \rightarrow$ $C$. We fix a minimal section $C_{0}$ such that $\mathcal{O}_{X}\left(C_{0}\right)=\mathcal{O}_{\mathbb{P}_{C}(\mathcal{E})}(1)$. For $\mathfrak{b} \in \operatorname{Pic} C, \mathfrak{b} f$ denote the pullback of $\mathfrak{b}$ by $\pi$. Thus any element of $\operatorname{Pic} X$ can be written $a C_{0}+\mathfrak{b} f$ with $a \in \mathbb{Z}$ and $\mathfrak{b} \in \operatorname{Pic} C$ and any element of $\operatorname{Num} X$ can be written $a C_{0}+b f$ with $a, b \in \mathbb{Z}$.

When $g=1$, it is proved by Yuko Homma [12, 13] and Gallego-Purnaprajna [3] that property $N_{0}$ and $N_{1}$ are characterized in terms of the intersection number of $L$ with a minimal section, a fiber and the anticanonical curve. See Remark 1.2 and 1.3 .

Theorem 1.2. Let $X$ be an elliptic ruled surface, and let $L \in$ PicX be a line bundle in the numerical class of $a C_{0}+b f$.

(1) (Yuko Homma, 12, 13]) If $e \geq 0$, then $L$ is normally generated if and only if $a \geq 1$ and $b-a e \geq 3$. If $e=-1$, then $L$ is normally generated if and only if $a \geq 1, a+b \geq 3$ and $a+2 b \geq 3$.

(2) (F. J. Gallego and B. P. Purnaprajna, 3]) If $e \geq 0$, then L satisfies property $N_{1}$ if and only if $a \geq 1$ and $b-a e \geq 4$. If $e=-1$, then $L$ satisfies property $N_{1}$ if and only if $a \geq 1, a+b \geq 4$ and $a+2 b \geq 4$. 
For higher syzygies, F. J. Gallego and B. P. Purnaprajna [4] obtained the following:

Theorem 1.3 (F. J. Gallego and B. P. Purnaprajna, [4]). Let $X$ be an elliptic ruled surface with the numerical invariant $e$.

(1) Let $L$ be a line bundle in the numerical class of $a C_{0}+b f$.

(a) If $e=-1$ and $a \geq p+1, a+b \geq 2 p+2$, and $a+2 b \geq 2 p+2$, then $L$ satisfies property $N_{p}$.

(b) If $e \geq 0$ and $a \geq p+1, b-a e \geq 2 p+2$, then $L$ satisfies property $N_{p}$.

(2) If $B_{1}, \cdots, B_{p+1} \in$ PicX are ample and base point free line bundles, then property $N_{p}$ holds for $B_{1}+\cdots+B_{p+1}$.

(3) If $A_{1}, \cdots, A_{2 p+3} \in P i c X$ are ample line bundles, then $K_{X}+A_{1}+\cdots+A_{2 p+3}$ satisfies property $N_{p}$.

They also conjectured the following:

Conjecture (F. J. Gallego and B. P. Purnaprajna, 4]). Let X be an elliptic ruled surface, and let $L \in P i c X$ be a line bundle in the numerical class $a C_{0}+b f$.

(1) If $e \geq 0$, then $L$ satisfies property $N_{p}$ if and only if $a \geq 1$ and $b-a e \geq 3+p$.

(2) If $e=-1$, then $L$ satisfies property $N_{p}$ if and only if $a \geq 1, a+b \geq 3+p$, and $a+2 b \geq p+3$.

Remark 1.1. When $e \geq 0$, $\operatorname{deg}\left(\left.L\right|_{C_{0}}\right)=L \cdot C_{0}=b-a e$. Also, $\left(C,\left.L\right|_{C_{0}}\right)$ satisfies property $N_{p}$ if and only if $\operatorname{deg}\left(\left.L\right|_{C_{0}}\right) \geq 3+p$ by Theorem 1.1. Therefore this conjecture suggests that $(X, L)$ satisfies property $N_{p}$ if and only if $\left(C,\left.L\right|_{C_{0}}\right)$ satisfies property $N_{p}$.

Remark 1.2. When $e=-1$, note that there exists a smooth elliptic curve $E \subset X$ such that $E \equiv 2 C_{0}-f$. See Proposition 3.2 in 3 . Also $\operatorname{deg}\left(\left.L\right|_{C_{0}}\right)=L \cdot C_{0}=a+b$ and $\operatorname{deg}\left(\left.L\right|_{E}\right)=L \cdot E=a+2 b$. Thus $\left(C,\left.L\right|_{C_{0}}\right)$ satisfies property $N_{p}$ if and only if $a+b \geq 3+p$, and $\left(E,\left.L\right|_{E}\right)$ satisfies property $N_{p}$ if and only if $a+2 b \geq 3+p$ by Theorem 1.1. Therefore this conjecture suggests that when $e=-1,(X, L)$ satisfies property $N_{p}$ if and only if $\left(C,\left.L\right|_{C_{0}}\right)$ and $\left(E,\left.L\right|_{E}\right)$ satisfy property $N_{p}$.

This conjecture has been solved for $p=0,1$ by Theorem 1.2 and our first main result is that this conjecture is true when $e \geq 0$ or $e=-1$ and $a=1$ :

Theorem 1.4. Let $X$ be an elliptic ruled surface, and let $L \in$ PicX be a line bundle in the numerical class $a C_{0}+b f$.

(1) If $e \geq 0$, then $L$ satisfies property $N_{p}$ if and only if $a \geq 1$ and $b-a e \geq 3+p$.

(2) If $e=-1$, then $L$ satisfies property $N_{p}$ if $a \geq 1$ and $a+2 b \geq 5+2 p$.

(3) If $e=-1$ and $a=1$, then $L$ satisfies property $N_{p}$ if and only if $b \geq 2+p$.

We remove the condition $a \geq p+1$ in Theorem 1.3. (1). Theorem 1.4 says that if $e \geq 0$ or if $e=-1$ and $a=1$, then $(X, L)$ satisfies property $N_{p}$ if and only if $\left(C, L_{C}\right)$ satisfies property $N_{p}$, where $L_{C}$ is the restriction of $L$ to $C_{0}$. In particular, property $N_{p}$ is characterized in terms of the intersection number of $L$ with a minimal section and a fiber. Also we classify all projectively normal elliptic surface scrolls which satisfy property $N_{p}$.

Corollary 1.5. Let $L=K_{X}+A_{1}+\cdots+A_{q}$, where $A_{i}$ is ample and $q \geq 3$. If $e \geq 0$, then $L$ satisfies property $N_{p}$ if $q \geq 3-e+p$. 
Corollary 1.6. Let $L=B_{1} \otimes \cdots \otimes B_{p+1}$ be a line bundle on $X$ such that each $B_{i}$ is ample and base point free and $p \geq 1$. If $e \geq 0$, then $L$ satisfies property $N_{2 p-1}$.

Corollaries 1.5 and 1.6 refine numerical bounds in Theorem 1.3(2) and (3) when $e \geq 0$. They are obtained by using Theorem 1.4 since all base point free line bundles and ample line bundles on $X$ are classified. Corollary 1.5 shows that Mukai's conjecture is true of elliptic ruled surfaces with $e \geq 0$. Also this is optimal. Indeed let $A=a C_{0}+b f$ be an ample line bundle such that $b-a e=1$. Then $K_{X}+(3+p-e) A$ fails to satisfy property $N_{p+1}$ by Theorem 1.4

Remark 1.3. Assume that $e=-1$ and let $A=a C_{0}+b f$ be an ample line bundle. If $a+2 b \geq 2$, i.e., $A$ is base point free, then $K_{X}+q A$ satisfies property $N_{p}$ for $q \geq 3+p$ since $K_{X}+q A=(a q-2) C_{0}+(b q+1) f$. Therefore for an elliptic ruled surface with $e=-1$, Mukai's conjecture is true for base point free ample line bundles, and the only remaining case is when $a+2 b=1$, that is, $A$ is ample but not base point free (e.g. $\left.D=C_{0}\right)$.

When $C$ is a curve of genus $g \geq 2$, the author has obtained that

Theorem 1.7 (Corollary 4.4, 14]). Let $X$ be a ruled surface over a curve $C$ of genus $g \geq 2$, and let $L \in P i c X$ be a line bundle in the numerical class of $a C_{0}+b f$.

(1) When $e \geq 0$, L satisfies property $N_{p}$ if $b-a e \geq 3 g-1+p$.

(2) When e $<0$, L satisfies property $N_{p}$ if $2 b-a e \geq 6 g-2+2 p$.

In this article we prove the following sharper result for $e \geq 0$ :

Theorem 1.8. Let $X$ be a ruled surface over a curve $C$ of genus $g \geq 2$. Let $L \in P i c X$ be a line bundle in the numerical class of $a C_{0}+b f$ and $L_{C}$ the restriction of $L$ to $C_{0}$.

(1) When $0 \leq e \leq g-3$, L satisfies property $N_{p}$ if $a \geq p+2$ and $b-a e \geq$ $3 g-1-e+p$.

(2) When $e \geq g-2, L$ satisfies property $N_{p}$ if $a \geq p+2$ and $b-a e \geq 2 g+1+p$.

(3) When $e \geq g-1$, assume that $a \geq p+3$ and $b-a e=2 g+p$. Then $(X, L)$ satisfies property $N_{p}$ if and only if $\left(C, L_{C}\right)$ satisfies property $N_{p}$.

Note that $\operatorname{deg}\left(L_{C}\right)=b-a e$. Therefore Theorem 1.1 and Theorem 1.8(3) guarantee the following:

(*) Let $L \in \operatorname{Pic} X$ be a line bundle in the numerical class of $a C_{0}+b f$ and let $L_{C}$ be the restriction of $L$ to $C_{0}$. When $e \geq g-1$ and $a \geq p+2$, if $b-a e=2 g+p$, then $(X, L)$ fails to satisfy property $N_{p}$ if and only if either

(i) $C$ is hyperelliptic or

(ii) $L_{C}=K_{C}+D$ for some effective divisor $D$ of degree $p+2$.

Example 1.1. Assume that $e \geq g-1$ and fix a positive integer $p$. Let $L=$ $a C_{0}+\mathfrak{b} f \in \operatorname{Pic} X$ be such that $a \geq p+3$ and

$$
\mathfrak{b}=K_{C}-a \mathfrak{e}+D,
$$

and $D$ is an effective divisor of degree $2+p$. Then by Theorem 1.1, $L_{C}=K_{C}+D$ does not satisfy Property $N_{p}$. So Theorem 1.8 implies that $(X, L)$ satisfies property $N_{p-1}$ and property $N_{p}$ does not hold.

Using Theorem 1.8 we prove the following Mukai type result. 
Corollary 1.9. Let $X$ be a ruled surface over a curve $C$ of genus $g \geq 2$ with $e \geq 0$. Let $L=K_{X}+A_{1}+\cdots+A_{q}$, where $A_{i}$ is ample.

(1) When $0 \leq e \leq g-3$, L satisfies property $N_{p}$ if $q \geq \max \{4, g+1-2 e\}+p$.

(2) When $e \geq g-2$, L satisfies property $N_{p}$ if $q \geq 4+p$.

In particular, Mukai's conjecture holds for $X$ if $e \geq \frac{g-3}{2}$.

Remark 1.4. Concerning Mukai's conjecture for ruled surfaces with arbitrary $e$, D. Butler's result 1 says that $K_{X}+(4+4 p) A$ satisfies property $N_{p}$. Also for ruled surfaces with $e \geq 0$, his work says that $a C_{0}+b f$ satisfies property $N_{p}$ if $a \geq p+1$ and $b-a e \geq 2 g+2 p$.

Remark 1.5. Let $X$ be a ruled surface over a curve of genus $g$. Corollary 1.9 shows that if $g=2,3$ and $e \geq 0$, then Mukai's conjecture holds for $X$.

To study the distribution of zeros in the Betti table, we use the so-called Koszul cohomology developed by Mark Green [8]. In particular, this method enables us to show some vanishing of Betti numbers by the vanishing of cohomology groups of certain vector bundles. We reduce the problem to show vanishing of cohomology groups on ruled surfaces to that on curves. Then we use some numerical conditions to kill higher cohomology groups of vector bundles on curves.

It seems the most interesting part of our result is that when $e \geq 0$, higher syzygies of ruled surfaces are closely related to those of the minimal section $C_{0}$. This follow from the long exact sequence of cohomology groups induced by the short exact sequence

$$
0 \rightarrow \mathcal{O}_{X}\left(-C_{0}\right) \rightarrow \mathcal{O}_{X} \rightarrow \mathcal{O}_{C} \rightarrow 0,
$$

associated to the minimal section $C_{0}$ on $X$. On a variety $Z$ and a line bundle $L \in \operatorname{Pic} Z$, a curve $C \subset Z$ is said to be extremal with respect to $L$ and property $N_{p}$ if $(X, L)$ and $\left(C, L_{C}\right)$ satisfy property $N_{p}$ but not property $N_{p+1}$. See Remark 1.5 in [7. When $X$ is a rational surface with anti-canonical divisor nef, F. J. Gallego and B. P. Purnaprajna [7] proved for any very ample $L \in \operatorname{Pic} X$, a smooth curve $C$ in $|L|$ is extremal with respect to $L$ by showing that the embedding of $X$ under $L$ is arithmetically Cohen Macaulay. Applying this to rational ruled surfaces, they obtained the following:

Theorem 1.10 (F. J. Gallego and B. P. Purnaprajna, 7]). Let X be the rational ruled surface associated to $\mathcal{O}_{\mathbb{P}^{1}} \oplus \mathcal{O}_{\mathbb{P}^{1}}(-e)$. Let $L$ be a line bundle in the numerical class of $a C_{0}+b f$.

(1) If $a=1$ or $e=0$ and $b=1$, then $L$ satisfies property $N_{p}$ for all $p \geq 0$.

(2) If $e=0$ and $a, b \geq 2$ or $e \geq 1$ and $a \geq 2$, then $L$ satisfies property $N_{p}$ if and only if $2 a+2 b-a e \geq 3+p$.

Unfortunately this does not hold for ruled surfaces over an irrational curve. More precisely, $h^{1}\left(X, \mathcal{O}_{X}\right)=g \geq 1$ and hence $(X, L)$ cannot be arithmetically Cohen Macaulay. So we prove a similar statement. Also, our results imply that for $e \geq 0$ the minimal section $C_{0}$ is the extremal curve. Obviously the existence of extremal curves enables us to apply fruitful results about higher syzygies of curves. When $e<0$, there may be more extremal curves. For an example, on an elliptic ruled surface $X$ with $e=-1$ there exists a smooth elliptic curve $E \subset X$ such that $E \equiv 2 C_{0}-f$. Theorems 1.2 and 1.4(3) make it affirmative that $C_{0}$ and $E$ are the extremal curves with respect to $L$ as conjectured by F. J. Gallego and B. P. Purnaprajna. 
The organization of this paper is as follows. In $\S 2$, we review some necessary elementary facts to study higher syzygies of ruled surfaces. $\S 3$ is devoted to give numerical criteria for Property $N_{p}$. In $\S 4$ we suggest some open questions related to our results.

\section{Preliminaries}

2.1. Notations and conventions. Throughout this paper the following is assumed.

(1) All varieties are defined over the complex number field $\mathbb{C}$.

(2) For a finite-dimensional $\mathbb{C}$-vector space $V, \mathbb{P}(V)$ is the projective space of one-dimensional quotients of $V$.

(3) When a variety $X$ is embedded in a projective space, we always assume that it is non-degenerate, i.e. it does not lie in any hyperplane.

(4) When a projective variety $X$ is embedded in a projective space $\mathbb{P}^{r}$ by a very ample line bundle $L \in \operatorname{Pic} X$, we may write $\mathcal{O}_{X}(1)$ instead of $L$ as long as there is no confusion.

2.2. The slope of vector bundles on a curve. Let $C$ be a smooth projective curve of genus $g$. For a vector bundle $\mathcal{F}$ on $C$, the slope $\mu(\mathcal{F})$ is defined by $\operatorname{deg}(\mathcal{F}) / \operatorname{rank}(\mathcal{F})$. Also the maximal slope and the minimal slope are defined as follows:

$$
\mu^{+}(\mathcal{F})=\max \{\mu(S) \mid 0 \rightarrow S \rightarrow \mathcal{F}\} \text { and } \mu^{-}(\mathcal{F})=\min \{\mu(Q) \mid \mathcal{F} \rightarrow Q \rightarrow 0\} .
$$

$\mathcal{F}$ is called semistable if $\mu(\mathcal{F})=\mu^{-}(\mathcal{F})$ and stable if $\mu(\mathcal{F})<\mu^{-}(\mathcal{F})$. These notions satisfy the following properties.

Lemma 2.1. For vector bundles $\mathcal{E}, \mathcal{F}$ and $\mathcal{G}$ on $C$,

(1) $\mu^{+}(\mathcal{E} \otimes \mathcal{F})=\mu^{+}(\mathcal{E})+\mu^{+}(\mathcal{F})$.

(2) $\mu^{-}(\mathcal{E} \otimes \mathcal{F})=\mu^{-}(\mathcal{E})+\mu^{-}(\mathcal{F})$.

(3) $\mu^{+}\left(S^{\ell}(\mathcal{E})\right)=\ell \mu^{+}(\mathcal{E})$.

(4) $\mu^{-}\left(S^{\ell}(\mathcal{E})\right)=\ell \mu^{-}(\mathcal{E})$.

(5) $\mu^{-}\left(\wedge^{\ell} \mathcal{E}\right) \geq \ell \mu^{-}(\mathcal{E})$.

(6) If $\mu^{-}(\mathcal{E})>2 g-2$, then $h^{1}(C, \mathcal{E})=0$.

(7) If $\mu^{-}(\mathcal{E})>2 g-1$, then $\mathcal{E}$ is globally generated.

(8) If $\mu^{-}(\mathcal{E})>2 g$, then $\mathcal{O}_{\mathbb{P}(\mathcal{E})}(1)$ is very ample.

(9) If $0 \rightarrow \mathcal{E} \rightarrow \mathcal{F} \rightarrow \mathcal{G} \rightarrow 0$ is an exact sequence, then

$$
\mu^{-}(\mathcal{G}) \geq \mu^{-}(\mathcal{F}) \geq \min \left\{\mu^{-}(\mathcal{E}), \mu^{-}(\mathcal{G})\right\} .
$$

Proof. See $\S 1$ and $\S 2$ in [1].

Therefore for a vector bundle $\mathcal{E}$, if $\mu^{-}(\mathcal{E})>2 g-1$, then the evaluation map determines an exact sequence of bundles:

$$
0 \rightarrow \mathcal{M}_{\mathcal{E}} \rightarrow H^{0}(C, \mathcal{E}) \otimes \mathcal{O}_{C} \rightarrow \mathcal{E} \rightarrow 0 .
$$

Butler obtained the following very useful result.

Theorem 2.2 (Butler, [1]). For a vector bundle $\mathcal{E}$ over $C$, if $\mu^{-}(\mathcal{E}) \geq 2 g$, then $\mathcal{M}_{\mathcal{E}}$ satisfies

$$
\mu^{-}\left(\mathcal{M}_{\mathcal{E}}\right) \geq-\frac{\mu^{-}(\mathcal{E})}{\mu^{-}(\mathcal{E})-g}
$$


2.3. Regularity of vector bundles over ruled varieties. Let $E$ be a vector bundle of rank $n+1$ over a smooth projective variety $Y$, and let $X=\mathbb{P}_{Y}(E)$ with the projection map $\pi: X \rightarrow Y$ and tautological line bundle $H$.

Definition 2.1. For a vector bundle $\mathcal{F}$ over $X$, we say that $\mathcal{F}$ is $f \pi$-regular when

$$
R^{i} \pi_{*}(\mathcal{F}(f-i))=0
$$

for every $i \geq 1$.

Here $\mathcal{F}(f-i)=\mathcal{F} \otimes H^{f-i}$. By definition, a line bundle of the form $a H+\pi^{*} B$ is $(-a) \pi$-regular. We present some basic facts about the $\pi$-regularity.

Lemma 2.3 (Lemma 3.2, [1]). Let $\mathcal{F}$ and $\mathcal{G}$ be two vector bundles on $X$ with $f$ and $g \pi$-regularity, respectively.

(1) $\mathcal{F} \otimes \mathcal{G}$ is $(f+g) \pi$-regular.

(2) If $f \leq 1$, then

$$
H^{i}(X, \mathcal{F}) \cong H^{i}\left(Y, \pi_{*} \mathcal{F}\right) \text { for all } i \geq 0 .
$$

(3) If $f \leq 0$ and $\widetilde{\mathcal{F}}=\pi^{*}\left(\pi_{*} \mathcal{F}\right)$, there is an exact sequence of vector bundles on $X$

$$
0 \rightarrow \mathcal{K}_{\widetilde{\mathcal{F}}} \rightarrow \widetilde{\mathcal{F}} \rightarrow \mathcal{F} \rightarrow 0
$$

where $\mathcal{K}_{\widetilde{\mathcal{F}}}$ is $1 \pi$-regular.

(4) If $f \leq 0$ and $g \leq 0$, then there is a surjective map

$$
\pi_{*} \mathcal{F} \otimes \pi_{*} \mathcal{G} \rightarrow \pi_{*}(\mathcal{F} \otimes \mathcal{G}) \rightarrow 0 .
$$

In particular if $Y$ is a curve, $\mu^{-}\left(\pi_{*}(\mathcal{F} \otimes \mathcal{G})\right) \geq \mu^{-}\left(\pi_{*} \mathcal{F}\right)+\mu^{-}\left(\pi_{*} \mathcal{G}\right)$.

2.4. Ruled surfaces. Let $X$ be a ruled surface over a smooth projective curve $C$ of genus $g$. We use notations in $\S 1$ and survey some basic facts about $X$ :

(1) The restriction of $a C_{0}+\mathfrak{b} f$ to $C_{0}$ is $\mathfrak{b}+a \mathfrak{e}$.

(2) The canonical line bundle of $X$ is given by $K_{X}=-2 C_{0}+\left(K_{C}+\mathfrak{e}\right) f$.

(3) The arithmetic genus $p_{a}(X)=-g$ and the geometric genus $p_{g}(X)=0$.

(4) When $e \geq 0, \mu^{-}(\mathcal{E})=-e$. When $e \leq-1, \mu^{-}(\mathcal{E})=-\frac{e}{2}$.

(5) $\mathcal{E}$ is nonstable if and only if $e>0$, semistable if and only if $e \leq 0$, and stable if and only if $e<0$.

(6) Let $L \in \operatorname{Pic} X$ be a line bundle in the numerical class of $a C_{0}+b f$.

(i) If $e \geq 0$, then $L$ is ample if and only if $a \geq 1$ and $b-a e \geq 1$.

(ii) If $e<0$, then $L$ is ample if and only if $a \geq 1$ and $2 b-a e \geq 1$.

2.5. Koszul cohomology. Let $V$ be a finite-dimensional complex vector space, and let $S(V)$ be the symmetric algebra on $V$. For a non-zero coherent sheaf $\mathcal{F}$ on $\mathbb{P}=\mathbb{P} V$, consider the associated graded $S$-module

$$
F=\bigoplus_{\ell \in \mathbb{Z}} H^{0}(\mathbb{P}, \mathcal{F}(\ell))
$$

and the minimal free resolution

$$
\begin{aligned}
& \cdots \rightarrow \bigoplus_{q \in \mathbb{Z}} S(V)(-q) \otimes M_{i, q} \stackrel{\varphi_{i}}{\rightarrow} \bigoplus_{q \in \mathbb{Z}} S(V)(-q) \otimes M_{i-1, q} \rightarrow \cdots \\
& \rightarrow \bigoplus_{q \in \mathbb{Z}} S(V)(-q) \otimes M_{1, q} \stackrel{\varphi_{1}}{\rightarrow} \rightarrow \bigoplus_{q \in \mathbb{Z}} S(V)(-q) \otimes M_{0, q} \stackrel{\varphi_{0}}{\rightarrow} F \rightarrow 0
\end{aligned}
$$


of $F$. Put $k_{i, j}=\operatorname{dim}_{k} M_{i, i+j}$. These integers are called Betti numbers of $F$. There is the following general connection between syzygies and some cohomology groups.

Theorem 2.4 (Theorem 4.5, [2]). There is an exact sequence

$$
\begin{aligned}
& \bigwedge^{i+1} V \otimes H^{0}(\mathbb{P}, \mathcal{F}(j-1)) \stackrel{\alpha_{i, j}}{\rightarrow} H^{0}\left(\mathbb{P}, \wedge^{i} \mathcal{M} \otimes \mathcal{F}(j)\right) \\
\rightarrow & H^{1}\left(\mathbb{P}, \bigwedge^{i+1} \mathcal{M} \otimes \mathcal{F}(j-1)\right) \rightarrow \bigwedge^{i+1} V \otimes H^{1}(\mathbb{P}, \mathcal{F}(j-1))
\end{aligned}
$$

with $\operatorname{Coker}\left(\alpha_{i, j}\right) \cong M_{i, i+j}$ where $\mathcal{M}=\Omega_{\mathbb{P} r}(1)$ and $V=H^{0}\left(\mathbb{P}^{r}, \mathcal{O}_{\mathbb{P}^{r}}(1)\right)$. Therefore $k_{i, j}=\operatorname{dim}_{k} \operatorname{Coker}\left(\alpha_{i, j}\right)$ and we have the exact sequence

$$
0 \rightarrow M_{i, i+j} \rightarrow H^{1}\left(\mathbb{P}, \bigwedge^{i+1} \mathcal{M} \otimes \mathcal{F}(j-1)\right) \rightarrow \bigwedge^{i+1} V \otimes H^{1}(\mathbb{P}, \mathcal{F}(j-1)) .
$$

In this paper, we concern ourselves with the case when $\mathcal{F}$ is a coherent sheaf on a subvariety $X \subset \mathbb{P}$. Under this situation, Theorem 2.4 guarantees that there is an exact sequence

$$
\begin{gathered}
\bigwedge^{i+1} V \otimes H^{0}(X, \mathcal{F}(j-1)) \stackrel{\alpha_{i, j}}{\rightarrow} H^{0}\left(X, \wedge^{i} \mathcal{M} \otimes \mathcal{F}(j)\right) \\
\rightarrow H^{1}\left(X, \bigwedge^{i+1} \mathcal{M} \otimes \mathcal{F}(j-1)\right) \rightarrow \bigwedge^{i+1} V \otimes H^{1}(X, \mathcal{F}(j-1))
\end{gathered}
$$

with $\operatorname{Coker}\left(\alpha_{i, j}\right) \cong M_{i, i+j}$.

2.6. Cohomological interpretation of property $N_{p}$. We review some cohomological criteria for property $N_{p}$. Let $X$ be a smooth projective variety of dimension $n \geq 1$, and let $L \in \operatorname{Pic} X$ be a very ample line bundle. Consider the natural short exact sequence

$$
0 \rightarrow \mathcal{M}_{L} \rightarrow H^{0}(X, L) \otimes \mathcal{O}_{X} \rightarrow L \rightarrow 0
$$

Lemma 2.5. Suppose that the ideal sheaf $\mathcal{I}_{X / \mathbb{P}}$ of $X \hookrightarrow \mathbb{P}=\mathbb{P} H^{0}(X, L)$ is 3 -regular in the sense of Castelnuovo-Mumford, i.e., that $H^{i}\left(\mathbb{P}, \mathcal{I}_{X / \mathbb{P}}(3-i)\right)=0$ for all $i \geq 1$. Then for $p \leq \operatorname{codim}(X, \mathbb{P})$, property $N_{p}$ holds for $L$ if and only if $H^{1}\left(X, \bigwedge^{p+1} \mathcal{M}_{L} \otimes L\right)=0$.

Proof. See $\S 1$ in [10].

Remark 1. Let $X$ be a smooth projective surface with geometric genus 0, i.e., $H^{2}\left(X, \mathcal{O}_{X}\right)=0$. Let $L \in \operatorname{Pic} X$ be a normally generated very ample line bundle such that $H^{1}(X, L)=0$. Then it is easy to check that $\mathcal{I}_{X / \mathbb{P}}$ is 3-regular.

2.7. Higher syzygies of degenerate varieties. Let $\Lambda \cong \mathbb{P} W \subset \mathbb{P} V$ be a linear subspace such that $\operatorname{codim}(W, V)=c$. It is easily checked that

$$
\left.\Omega_{\mathbb{P} V}(1)\right|_{\mathbb{P} W} \cong \Omega_{\mathbb{P} W}(1) \oplus \mathcal{O}_{\mathbb{P} W}^{c} .
$$

Now let $X \subset \mathbb{P} V$ be a smooth projective variety which is indeed contained in $\Lambda$. Let the corresponding very ample line bundle on $X$ be $L \in \operatorname{Pic} X$. Consider the natural short exact sequences

$$
\begin{gathered}
0 \rightarrow \mathcal{M}_{V} \rightarrow V \otimes \mathcal{O}_{X} \rightarrow L \rightarrow 0 \quad \text { and } \\
0 \rightarrow \mathcal{M}_{W} \rightarrow W \otimes \mathcal{O}_{X} \rightarrow L \rightarrow 0 .
\end{gathered}
$$

The above observation shows that $\mathcal{M}_{V} \cong \mathcal{M}_{W} \oplus \mathcal{O}_{X}^{c}$. 
Lemma 2.6. Under the situation just stated, assume that $H^{1}\left(X, L^{\ell}\right)=0$ for all $\ell \geq 1$ and $W=H^{0}(X, L)$. Then $(X, L)$ satisfies property $N_{p}$ if and only if

$$
H^{1}\left(X, \bigwedge^{i} \mathcal{M}_{V} \otimes L^{\ell}\right)=0 \text { for } 1 \leq i \leq p+1 \text { and } \ell \geq 1 \text {. }
$$

Proof. We use induction on $c$. When $c=0$, this is a well-known criterion for Property $N_{p}$.

Assume that $c=1$. Since $\mathcal{M}_{W}=\mathcal{M}_{V} \oplus \mathcal{O}_{X}, \bigwedge^{i} \mathcal{M}_{V} \cong \bigwedge^{i} \mathcal{M}_{W} \oplus \bigwedge^{i-1} \mathcal{M}_{W}$, which completes the proof.

When $c \geq 2$, fix a filtration $H^{0}(X, L)=W \subset W_{1} \subset \cdots \subset W_{c}=V$ of subspaces each having codimension one in the next. Then assertion comes by repeating the process in the case $c=1$.

\section{MAIN THEOREMS}

Let $\pi: X \rightarrow C$ be the projection morphism. Let $L=a C_{0}+\mathfrak{b} f \in \operatorname{Pic} X$ be a line bundle in the numerical class of $a C_{0}+b f$ such that $a \geq 1$ and $b+a \mu^{-} *(\mathcal{E}) \geq 2 g+1$. Let $\mathcal{F}$ denote $\pi_{*} L=S^{a}(\mathcal{E}) \otimes \mathfrak{b}$. Then $\mu^{-}\left(\pi_{*} L\right)=b+a \mu^{-} *(\mathcal{E})$ and hence by Lemma 2.1. $L$ is very ample and $H^{1}\left(X, L^{\ell}\right)=0$ if $\ell \neq 0$. Now consider the exact sequences

$$
\begin{aligned}
0 & \rightarrow \mathcal{M}_{\mathcal{F}} \rightarrow H^{0}(C, \mathcal{F}) \otimes \mathcal{O}_{C} \rightarrow \mathcal{F} \rightarrow 0 \quad \text { and } \\
0 & \rightarrow \mathcal{M}_{L} \rightarrow H^{0}(X, L) \otimes \mathcal{O}_{X} \rightarrow L \rightarrow 0 .
\end{aligned}
$$

Lemma 3.1. Let $N \in P i c X$ be a line bundle in the numerical class of $s C_{0}+t f$.

(1) For $m \geq 1, H^{1}\left(X, \bigwedge^{m} \mathcal{M}_{L} \otimes N\right)=0$ if

$$
s \geq \min \{m, a\} \text { and } t+s \mu^{-}(\mathcal{E})>\frac{m\left(b+a \mu^{-}(\mathcal{E})\right)}{b+a \mu^{-}(\mathcal{E})-g}+2 g-2 .
$$

(2) For $m \geq 1, H^{2}\left(X, \bigwedge^{m} \mathcal{M}_{L} \otimes N\right)=0$ if $s+1 \geq m$.

Proof. Consider the exact sequence

$$
0 \rightarrow \mathcal{K}_{L} \rightarrow \pi^{*} \mathcal{F} \rightarrow L \rightarrow 0
$$

where $\mathcal{K}_{L}$ is a vector bundle of rank $a$ on $X$ which is $1 \pi$-regular by Lemma 2.3. Using the Snake Lemma, we have the following commutative diagram:

0
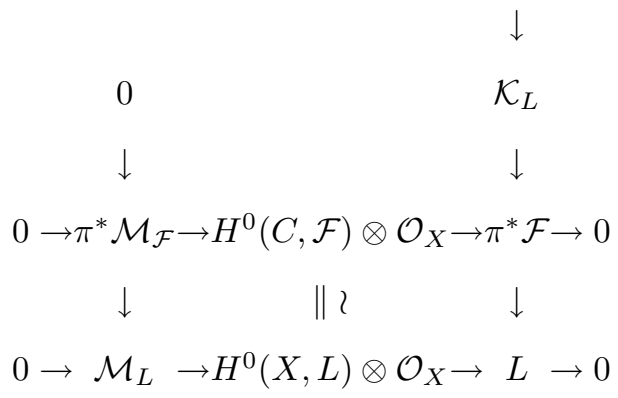

$\mathcal{K}_{L}$

0 
(1) From the exact sequence

$$
0 \rightarrow \pi^{*} \mathcal{M}_{\mathcal{F}} \rightarrow \mathcal{M}_{L} \rightarrow \mathcal{K}_{L} \rightarrow 0,
$$

the desired vanishing is obtained if

$$
H^{1}\left(X, \bigwedge^{m-i} \pi^{*} \mathcal{M}_{\mathcal{F}} \otimes \bigwedge^{i} \mathcal{K}_{L} \otimes N\right)=0 \text { for all } 0 \leq i \leq \min \{m, a\} .
$$

Since we are in characteristic zero, $\bigwedge^{i} \mathcal{K}_{L}$ is a direct summand of $T^{i} \mathcal{K}_{L}$, and therefore it suffices to show that

$$
H^{1}\left(X, \bigwedge^{m-i} \pi^{*} \mathcal{M}_{\mathcal{F}} \otimes T^{i} \mathcal{K}_{L} \otimes N\right)=0 \text { for all } 0 \leq i \leq \min \{m, a\} .
$$

Lemma 2.3 guarantees that $T^{i} \mathcal{K}_{L}$ is $i \pi$-regular and hence

$$
H^{1}\left(X, \bigwedge^{m-i} \pi^{*} \mathcal{M}_{\mathcal{F}} \otimes T^{i} \mathcal{K}_{L} \otimes N\right)=H^{1}\left(C, \bigwedge^{m-i} \mathcal{M}_{\mathcal{F}} \otimes \pi_{*} T^{i} \mathcal{K}_{L} \otimes N\right)
$$

from the assumption $s \geq \min \{m, a\}$. So by Lemma 2.1, it suffices to show that

$$
\mu^{-}\left(\bigwedge^{m-i} \mathcal{M}_{\mathcal{F}} \otimes \pi_{*} T^{i} \mathcal{K}_{L} \otimes N\right)>2 g-2
$$

First we claim that

(i) $\mu^{-}\left(\pi_{*} \mathcal{K}_{L} \otimes C_{0}\right) \geq b+a \mu^{-}(\mathcal{E})$.

(ii) $\mu^{-}\left(\pi_{*} T^{i} \mathcal{K}_{L} \otimes N\right) \geq i\left(b+a \mu^{-}(\mathcal{E})\right)+t+s \mu^{-}(\mathcal{E})$.

For (i), apply Lemma 4.3 in [1] to the case $V=L$ and $W=C_{0}$ in Butler's notation.

Since $\mathcal{K}_{L} \otimes C_{0}$ is $0 \pi$-regular and

$$
T^{i} \mathcal{K}_{L} \otimes N=T^{i}\left(\mathcal{K}_{L} \otimes C_{0}\right) \otimes\left((s-i) C_{0}+t f\right),
$$

repeated application of Lemma 2.3(4) shows that

$$
\begin{aligned}
\mu^{-}\left(\pi_{*} T^{i} \mathcal{K}_{L} \otimes N\right) & \geq i \mu^{-}\left(\pi_{*} \mathcal{K}_{L} \otimes C_{0}\right)+\mu^{-}\left(\pi_{*}\left\{(s-i) C_{0}+t f\right\}\right) \\
& \geq i\left(b+(a+1) \mu^{-}(\mathcal{E})\right)+(s-i) \mu^{-}(\mathcal{E})+t \\
& =i\left(b+a \mu^{-}(\mathcal{E})\right)+t+s \mu^{-}(\mathcal{E}),
\end{aligned}
$$

which completes the proof of (ii). From this claim,

$$
\begin{aligned}
\mu^{-}\left(\bigwedge^{m-i} \mathcal{M}_{\mathcal{F}} \otimes \pi_{*} T^{i} \mathcal{K}_{L} \otimes N\right) & \geq(m-i) \mu^{-}\left(\mathcal{M}_{\mathcal{F}}\right)+i\left(b+a \mu^{-}(\mathcal{E})\right)+t+s \mu^{-}(\mathcal{E}) \\
& \geq m \mu^{-}\left(\mathcal{M}_{\mathcal{F}}\right)+t+s \mu^{-}(\mathcal{E}) \\
& \geq-m \frac{\mu^{-}(\mathcal{F})}{\mu^{-}(\mathcal{F})-g}+t+s \mu^{-}(\mathcal{E}) \text { (Theorem 2.2). }
\end{aligned}
$$

Since $\mu^{-}(\mathcal{F})=b+a \mu^{-}(\mathcal{E})$,

$$
-\frac{m\left(b+a \mu^{-}(\mathcal{E})\right)}{b+a \mu^{-}(\mathcal{E})-g}+t+s \mu^{-}(\mathcal{E})>2 g-2
$$

implies the desired vanishing.

(2) In the same way, $H^{2}\left(X, \bigwedge^{m} \mathcal{M}_{L} \otimes N\right)=0$ if

$$
H^{2}\left(X, \bigwedge^{m-i} \pi^{*} \mathcal{M}_{\mathcal{F}} \otimes T^{i} \mathcal{K}_{L} \otimes N\right)=0 \text { for all } 0 \leq i \leq m .
$$

Since $T^{i} \mathcal{K}_{L}$ is $i \pi$-regular, if $s+1 \geq m$, then $T^{i} \mathcal{K}_{L} \otimes N$ is $1 \pi$-regular and hence

$$
H^{2}\left(X, \bigwedge^{m-i} \pi^{*} \mathcal{M}_{\mathcal{F}} \otimes T^{i} \mathcal{K}_{L} \otimes N\right)=H^{2}\left(C, \bigwedge^{m-i} \mathcal{M}_{\mathcal{F}} \otimes \pi_{*} T^{i} \mathcal{K}_{L} \otimes N\right)=0
$$

by Lemma 2.3 . 
Proof of Theorem 1.4(1) and (2). By Lemma 3.1. $H^{1}\left(X, \wedge^{m} \mathcal{M}_{L} \otimes L\right)=0$ if

$$
b+a \mu^{-}(\mathcal{E})>\frac{m\left(b+a \mu^{-}(\mathcal{E})\right)}{b+a \mu^{-}(\mathcal{E})-1}
$$

or equivalently $b+a \mu^{-}(\mathcal{E})>m+1$. Therefore if $b+a \mu^{-}(\mathcal{E})>2+p$, then $L$ satisfies property $N_{p}$ by Lemma 2.5] and Remark 11. Since $\mu^{-}(\mathcal{E})=-e$ if $e \geq 0$ and $\mu^{-}(\mathcal{E})=\frac{1}{2}$ if $e=-1$, it is proved that

(1) If $e \geq 0$, then $L$ satisfies property $N_{p}$ if $a \geq 1$ and $b-a e \geq 3+p$.

(2) If $e=-1$, then $L$ satisfies property $N_{p}$ if $a \geq 1$ and $a+2 b \geq 5+2 p$.

To complete the proof it remains to show that when $e \geq 0$,

(*) if $b-a e=3+p$ for some $p \geq 0$, then $L$ fails to satisfy property $N_{p+1}$.

Consider the exact sequence

$$
0 \rightarrow \mathcal{O}_{X}\left(-C_{0}\right) \rightarrow \mathcal{O}_{X} \rightarrow \mathcal{O}_{C} \rightarrow 0 .
$$

Define graded $S$-modules $R_{1}=\bigoplus_{\ell \in \mathbb{Z}} H^{0}\left(X, \mathcal{O}_{X}\left(-C_{0}\right) \otimes L^{\ell}\right), R_{2}=\bigoplus_{\ell \in \mathbb{Z}} H^{0}\left(X, L^{\ell}\right)$, and $R_{3}=\bigoplus_{\ell \in \mathbb{Z}} H^{0}\left(C, L_{C}^{\ell}\right)$, where $L_{C}$ is the restriction of $L$ to $C$. It is easy to check that $H^{1}\left(X, \mathcal{O}_{X}\left(-C_{0}\right) \otimes L^{\ell}\right)=0$ for all $\ell>0$ (see Proposition 3.1 in [3]). So we have the exact sequence

$$
0 \rightarrow R_{1} \rightarrow R_{2} \rightarrow R_{3} \rightarrow 0
$$

of graded $S$-modules with maps preserving the gradings and hence there is a long exact sequence

$$
\begin{array}{r}
\quad \cdots \rightarrow M_{1, q}\left(R_{1}, V\right) \rightarrow M_{1, q}\left(R_{2}, V\right) \rightarrow M_{1, q}\left(R_{3}, V\right) \\
\rightarrow M_{0, q}\left(R_{1}, V\right) \rightarrow M_{0, q}\left(R_{2}, V\right) \rightarrow M_{0, q}\left(R_{3}, V\right) \rightarrow 0 .
\end{array}
$$

See Corollary (1.d.4) in [8]. We need the following part:

$$
\cdots \rightarrow M_{p+1, p+3}\left(R_{2}, V\right) \rightarrow M_{p+1, p+3}\left(R_{3}, V\right) \rightarrow M_{p, p+3}\left(R_{1}, V\right) \rightarrow \cdots .
$$

Since $H^{1}\left(X, \mathcal{O}_{X}\left(-C_{0}\right) \otimes L^{2}\right)=0$,

$$
M_{p, p+3}\left(R_{1}, V\right)=H^{1}\left(X, \bigwedge^{p+1} \mathcal{M}_{L} \otimes \mathcal{O}_{X}\left(-C_{0}\right) \otimes L^{2}\right)
$$

by Theorem 2.4. By Lemma 3.1, $H^{1}\left(X, \bigwedge^{p+1} \mathcal{M}_{L} \otimes \mathcal{O}_{X}\left(-C_{0}\right) \otimes L^{2}\right)=0$, and hence $M_{p, p+3}\left(R_{1}, V\right)=0$.

Assume that $L$ satisfies property $N_{p+1}$. Then $M_{p+1, p+3}\left(R_{2}, V\right)=0$ and hence $M_{p+1, p+3}\left(R_{3}, V\right)=0$ which implies that $\left(C, L_{C}\right)$ satisfies property $N_{p+1}$. This is impossible since $\operatorname{deg}\left(L_{C}\right)=3+p$. So $M_{p+1, p+3}\left(R_{2}, V\right) \neq 0$ and hence property $N_{p+1}$ does not hold for $(X, L)$.

Now we prove Corollaries 1.5 and 1.6, Recall numerical criteria for base point freeness for line bundles on $X$.

Lemma 3.2. Let $L \in P i c X$ be a line bundle in the numerical class of $a C_{0}+b f$.

(1) If $e \geq 0$, then $L$ is ample and base point free if and only if $a \geq 1$ and $b-a e \geq 2$.

(2) If $e=-1$, then $L$ is ample and base point free if and only if $a \geq 1, a+b \geq 2$ and $a+2 b \geq 2$.

Proof. See Remark 3.5.3 in [3]. 
Proof of Corollary 1.5, Put $A_{i} \equiv a_{i} C_{0}+b_{i} f$. Thus $a_{i} \geq 1$ and $b_{i}-a_{i} e \geq 1$ by Lemma 3.2 Also,

$$
K_{X}+A_{1}+\cdots+A_{q} \equiv\left(\sum a_{i}-2\right) C_{0}+\left(\sum b_{i}-e\right) f
$$

Since $\left(\sum b_{i}-e\right)-e\left(\sum a_{i}-2\right)=\sum\left(b_{i}-a_{i} e\right)+e \geq q+e$ for $e \geq 0$, Theorem 1.4 guarantees (1).

Proof of Corollary 1.6. Put $B_{i}=a_{i} C_{0}+b_{i} f$. Thus $a_{i} \geq 1$ and $b_{i}-a_{i} e \geq 2$ by Lemma 3.2. Also

$$
L=\left(\sum a_{i}\right) C_{0}+\left(\sum b_{i}\right) f \text { and } \sum b_{i}-e \sum a_{i}=\sum\left(b_{i}-e a_{i}\right) \geq 2 p+2 .
$$

Then the assertion comes from Theorem 1.4 .

For a given normally generated very ample line bundle, one of the most natural questions to ask is: for which $p$ does the line bundle satisfy property $N_{p}$ ? If $X$ is an elliptic ruled surface with $e \geq 0$, this question is answered perfectly in Theorem 1.4(1). It seems very interesting that

(*) for a line bundle $L \in \operatorname{Pic} X$ in the numerical class of $a C_{0}+b f$ with $a \geq 1$, property $N_{p}$ holds for $L$ if and only if property $N_{p}$ holds for the restriction of $L$ to the minimal section $C_{0}$.

That is, for elliptic ruled surfaces with $e \geq 0$ the higher syzygies of line bundles are closely connected with that of the minimal section. Now we turn to the case $e=-1$. Recall that if $X$ is an elliptic ruled surface with $e=-1$, then there exists a smooth elliptic curve $E \subset X$ such that $E \equiv 2 C_{0}-f$. It is proved by Yuko Homma [12, [13 and Gallego-Purnaprajna [3] that property $N_{0}$ and $N_{1}$ are characterized in terms of the intersection number of $L$ with a minimal section, a fiber and the anticanonical curve $E$. Theorem 1.2 makes the following conjecture affirmative.

Conjecture (F. J. Gallego and B. P. Purnaprajna, 4]). Let $X$ be an elliptic ruled surface with $e=-1$ and let $L \in P i c X$ be a line bundle in the numerical class $a C_{0}+b f$. Then $L$ satisfies property $N_{p}$ if and only if $a \geq 1, a+b \geq 3+p$, and $a+2 b \geq p+3$.

For $p=2$, this is checked for $L$ in the numerical class $C_{0}+4 f$ or $2 C_{0}+3 f$ ([4], $\S 7)$. Now we show that this conjecture is true for scrolls, i.e., $a=1$.

Proof of Theorem 1.4(3). If $b \geq p+2$, then $L$ satisfies property $N_{p}$ by Theorem 1.4(2). For the converse, we prove a stronger statement:

(*) If $1 \leq a \leq p+3$ and $b=p+3-a$, then the line bundle $N \in \operatorname{Pic} X$ in the numerical class $a C_{0}+b f$ does not satisfy property $N_{p+1}$.

From the short exact sequence

$$
0 \rightarrow \mathcal{O}_{X}\left(-C_{0}\right) \rightarrow \mathcal{O}_{X} \rightarrow \mathcal{O}_{C} \rightarrow 0
$$

we obtain

$$
\begin{aligned}
\cdots \rightarrow H^{1}\left(X, \bigwedge^{p+2} \mathcal{M}_{N} \otimes N\right) & \rightarrow H^{1}\left(C, \bigwedge^{p+2} \mathcal{M}_{N} \otimes N_{C}\right) \\
& \rightarrow H^{2}\left(X, \bigwedge^{p+2} \mathcal{M}_{N} \otimes \mathcal{O}_{X}\left(-C_{0}\right) \otimes N\right) \rightarrow \cdots,
\end{aligned}
$$

where $N_{C}$ is the restriction of $N$ to $C_{0}$. Note that

(i) Since $\operatorname{deg}\left(N_{C}\right)=a+b=p+3, N_{C}$ fails to satisfy property $N_{p+1}$ and hence $H^{1}\left(C, \bigwedge^{p+2} \mathcal{M}_{N} \otimes N_{C}\right) \neq 0$ by Lemma 2.6. 


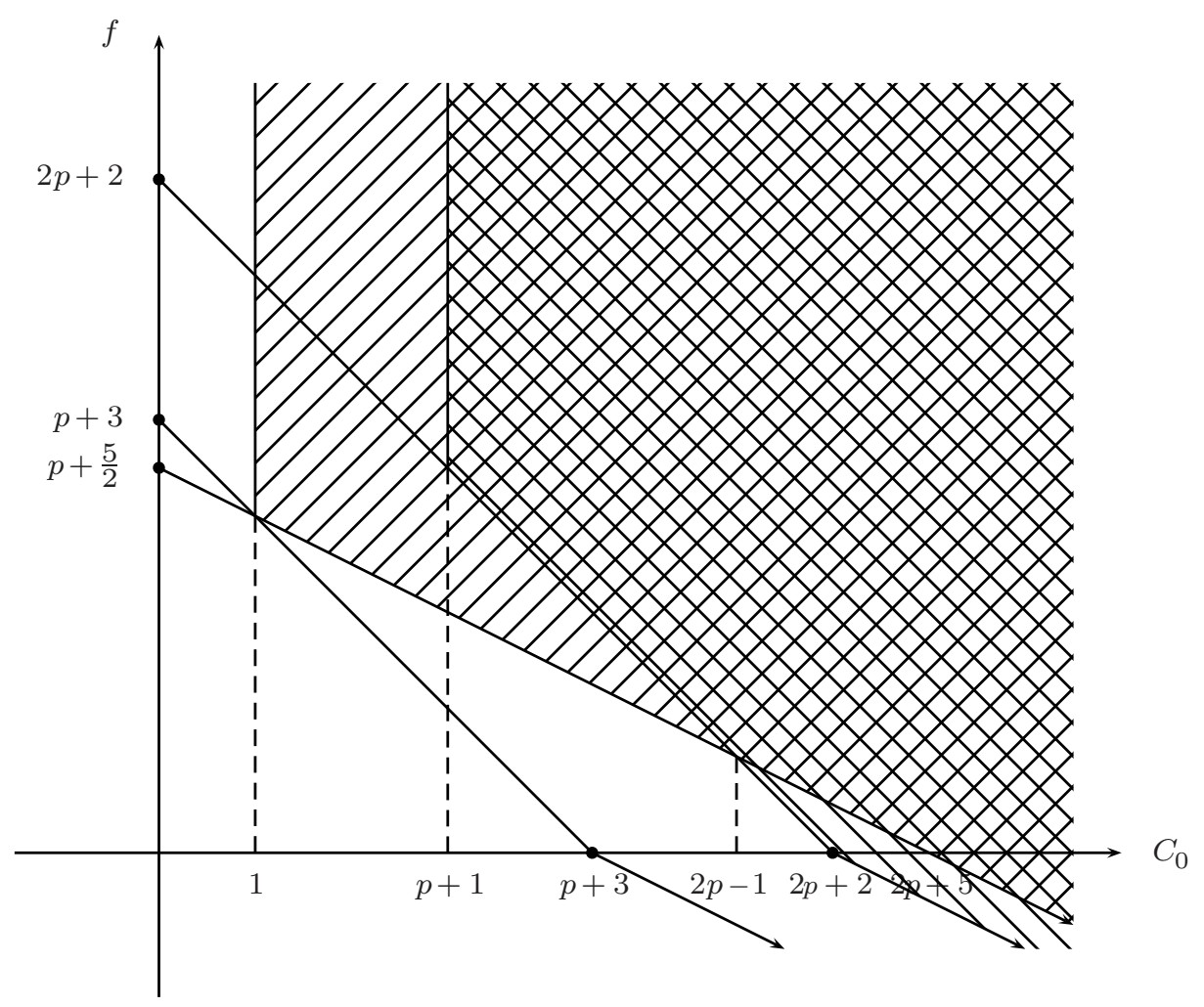

FiguRE 1. Elliptic ruled surface with $e=-1$

(ii) The short exact sequence

$$
0 \rightarrow \mathcal{M}_{N} \rightarrow H^{0}(X, N) \otimes \mathcal{O}_{X} \rightarrow N \rightarrow 0,
$$

gives the following long exact sequence:

$$
\begin{aligned}
\cdots \rightarrow H^{1}\left(X, \bigwedge^{p+1} \mathcal{M}_{N} \otimes \mathcal{O}_{X}\right. & \left.\left(-C_{0}\right) \otimes N^{2}\right) \rightarrow H^{2}\left(X, \bigwedge^{p+2} \mathcal{M}_{N} \otimes \mathcal{O}_{X}\left(-C_{0}\right) \otimes N\right) \\
& \rightarrow \bigwedge^{p+2} H^{0}(X, N) \otimes H^{2}\left(X, \mathcal{O}_{X}\left(-C_{0}\right) \otimes N\right) \rightarrow \cdots
\end{aligned}
$$

It is clear that $H^{2}\left(X, \mathcal{O}_{X}\left(-C_{0}\right) \otimes N\right)=0$. Also by Lemma 3.1 .

$$
H^{1}\left(X, \bigwedge^{p+1} \mathcal{M}_{N} \otimes \mathcal{O}_{X}\left(-C_{0}\right) \otimes N^{2}\right)=0
$$

and hence $H^{2}\left(X, \bigwedge^{p+2} \mathcal{M}_{N} \otimes \mathcal{O}_{X}\left(-C_{0}\right) \otimes N\right)=0$.

By (i) and (ii), $H^{1}\left(X, \bigwedge^{p+2} \mathcal{M}_{N} \otimes N\right) \neq 0$, which completes the proof.

Results on higher syzygies of elliptic ruled surfaces with $e=-1$ are summarized in Figure 1. In this figure, the integral points of the coordinate plane represent the classes of $\operatorname{Num}(X)$. Theorems 1.3 and 1.4 imply that the line bundles contained in the upper right area of the straight line or the higher crooked line should satisfy property $N_{p}$. The above conjecture by F. J. Gallego and B. P. Purnaprajna suggest that the line bundles contained in the right upper regions of the lower crooked line should satisfy property $N_{p}$. 
Proof of Theorem 1.8. From the short exact sequence

$$
0 \rightarrow \mathcal{O}_{X}\left(-C_{0}\right) \rightarrow \mathcal{O}_{X} \rightarrow \mathcal{O}_{C} \rightarrow 0,
$$

we obtain

$$
\begin{aligned}
\cdots & \rightarrow H^{1}\left(X, \bigwedge^{j} \mathcal{M}_{L} \otimes \mathcal{O}_{X}\left(-C_{0}\right) \otimes L\right) \rightarrow H^{1}\left(X, \bigwedge^{j} \mathcal{M}_{L} \otimes L\right) \\
& \rightarrow H^{1}\left(C, \bigwedge^{j} \mathcal{M}_{L} \otimes L_{C}\right) \rightarrow H^{2}\left(X, \bigwedge^{j} \mathcal{M}_{L} \otimes \mathcal{O}_{X}\left(-C_{0}\right) \otimes L\right) \rightarrow \cdots .
\end{aligned}
$$

Note that

(i) Since $\operatorname{deg}\left(L_{C}\right)=b-a e \geq 2 g+1+p, H^{1}\left(C, \bigwedge^{j} \mathcal{M}_{L} \otimes L \otimes \mathcal{O}_{C}\right)=0$ for all $1 \leq j \leq p+1$ by Lemma 2.6.

(ii) By Lemma 3.1, $H^{1}\left(X, \bigwedge^{j} \mathcal{M}_{L} \otimes \mathcal{O}_{X}\left(-C_{0}\right) \otimes L\right)=0$ for $1 \leq j \leq p+1$ if

$$
a-1 \geq p+1 \text { and } b-(a-1) e>\frac{(p+1)(b-a e)}{b-a e-g}+2 g-2 .
$$

Let $\nu=b-a e$. Then the second inequality is equivalent to

$$
\nu^{2}-(3 g-e-1+p) \nu+\left(2 g^{2}-2 g-e g\right)>0,
$$

and it is a tedious calculation that this inequality holds if $0 \leq e \leq g-3$ and $b-a e \geq 3 g-1-e+p$ or if $e \geq g-2$ and $b-a e \geq 2 g+1+p$.

(iii) By the same way as in (ii), when $e \geq g-1$,

$$
H^{1}\left(X, \bigwedge^{p+2} \mathcal{M}_{L} \otimes \mathcal{O}_{X}\left(-C_{0}\right) \otimes L\right)=0
$$

if $a \geq p+3$ and $b-a e \geq 2 g+1+p$.

(iv) By Lemma 3.1. $H^{2}\left(X, \wedge^{j} \mathcal{M}_{L} \otimes \mathcal{O}_{X}\left(-C_{0}\right) \otimes L\right)=0$ if $j \leq a$.

By (i) and (ii), $H^{1}\left(X, \wedge^{j} \mathcal{M}_{L} \otimes L\right)=0$ for all $1 \leq j \leq p+1$, which implies that $(X, L)$ satisfies property $N_{p}$ by Lemma 2.5. Therefore (1) and (2) are proved. By (iii) and (iv), $H^{1}\left(X, \bigwedge^{p+2} \mathcal{M}_{L} \otimes L\right) \cong H^{1}\left(C, \bigwedge^{p+2} \mathcal{M}_{L} \otimes L_{C}\right)$, which implies (3).

Proof of Corollary 1.9, Put $A_{i}=a_{i} C_{0}+b_{i} f$. Since $e \geq 0, A_{i}$ is ample if and only if $a_{\geq} 1$ and $b_{i}-a_{i} e \geq 1$. Let $L=K_{X}+A_{1}+\cdots+A_{q}$, which is in the numerical class of $\left(\sum a_{i}-2\right) C_{0}+\left(\sum b_{i}+2 g-2-e\right) f$. Note that

$$
\begin{aligned}
\left(\sum b_{i}+2 g-2-e\right)-e\left(\sum a_{i}-2\right) & =\sum\left(b_{i}-e a_{i}\right)+2 g-2+e \\
& \geq q+2 g-2+e .
\end{aligned}
$$

To apply Theorem 1.8, it is necessary that $\sum a_{i}-2 \geq p+2$, which is true since we assume that $q \geq 4+p$. Therefore $L$ satisfies property $N_{p}$ if

(1) $q+2 g-2+e \geq 3 g-1-e+p$ when $0 \leq e \leq g-3$, and

(2) $q+2 g-2+e \geq 2 g+1+p$ when $e \geq g-2$ or equivalently

$(1)^{\prime} q \geq \max \{g+1-2 e, 4\}+p$ when $0 \leq e \leq g-3$, and

$(2)^{\prime} q \geq 3-e+p$ when $e \geq g-2$.

Combining the assumption $q \geq 4+p$ and $(1)^{\prime},(2)^{\prime}$, we get the desired statement. 


\section{Open questions AND CONJECTURES}

For a given normally generated very ample line bundle, it is one of the most natural questions to ask for which $p$ does the line bundle satisfy property $N_{p}$ ? Along this line, we suggest open questions related to higher syzygies of line bundles on ruled surfaces. Let $X$ be a ruled surface over a smooth projective curve $C$ of genus $g$. Let $L \in \operatorname{Pic} X$ be a line bundle in the numerical class of $a C_{0}+b f$ with $a \geq 1$ and let $L_{C}$ be the restriction of $L$ to $C_{0}$.

4.1. Semistable or unstable cases. When $e \geq 0$, the following are known:

(1) (Theorem 1.2, 14]) If $b-a e \geq 3 g-1+p$, then property $N_{p}$ holds for $L$.

(2) (Theorem $1.8(1)$ ) When $0 \leq e \leq g-2, L$ satisfies property $N_{p}$ if $a \geq p+2$ and $b-a e \geq 3 g-1-e+p$.

(3) (Theorem $1.8(2)$ ) When $e \geq g-1, L$ satisfies property $N_{p}$ if $a \geq p+2$ and $b-a e \geq 2 g+1+p$.

These facts are summarized in Figure 1. In this figure, the integral points of the coordinate plane represent the classes of $\operatorname{Num}(X)$, and the shadowed regions contain the line bundles which satisfy property $N_{p}$. From these results and Green's " $2 g+1+p$ " theorem, the most natural hope in general would be the following:

Conjecture 1. L satisfies property $N_{p}$ if $b-a e \geq 2 g+1+p$.

This conjecture implies Mukai's conjecture for ruled surfaces with $e \geq 0$ as in the case of Corollary 1.9 (2). More precisely, this guarantees the following:

(*) Let $L=K_{X}+A_{1}+\cdots+A_{q}$, where $A_{i}$ is ample and $q \geq 3$. Then $L$ satisfies property $N_{p}$ if $q \geq 3-e+p$.

Also in the direction of Theorem $1.8(3)$, we make the following conjecture.

Conjecture 2. When $b-a e=2 g+p, L$ fails to satisfy property $N_{p}$ if and only if either

(i) $C$ is hyperelliptic or

(ii) $L_{C}=K_{C}+D$ for some effective divisor $D$ of degree $p+2$.

These two conjectures imply that when $e \geq 0$, the higher syzygies of line bundles would be closely connected with that of the minimal section. Theorem 1.4 implies exactly that these are true for elliptic ruled surfaces.

4.2. Stable cases. When $e<0$, it is known that

(*) (Theorem 1.2, [14]) If $2 b-a e \geq 6 g-2+2 p$, then Property $N_{p}$ holds for $L$. Note that when $e<0$, there may be other extremal curves as discussed in $\S 3$. Indeed, results of Yuko Homma [13] and Gallego-Purnaprajna 4] about elliptic ruled surfaces with $e=-1$ make the following conjecture affirmative.

Conjecture 3. L satisfies property $N_{p}$ if $b-a e \geq 2 g+1+p$ and $2 b-a e \geq 2 g+1+p$.

We remark that this is proved for $g=1$ and $a=1$, i.e., elliptic surface scrolls. As in the case $e \geq 0$, this guarantees Mukai's conjecture for ruled surfaces $e<0$. In Figure 2, the integral points of the coordinate plane represent the classes of $\operatorname{Num}(X)$, and the shadowed regions contain the line bundles which satisfy property $N_{p}$ 


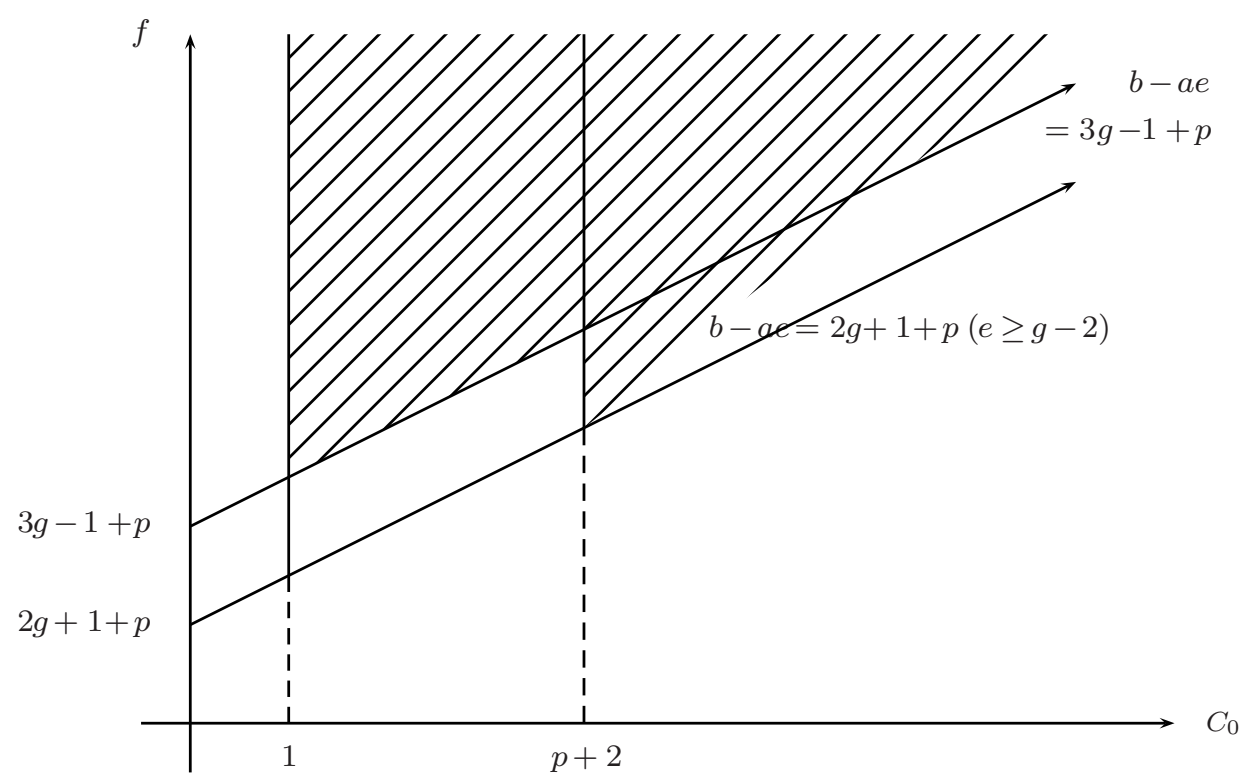

FiguRE 2. Ruled surface with $e \geq 0$

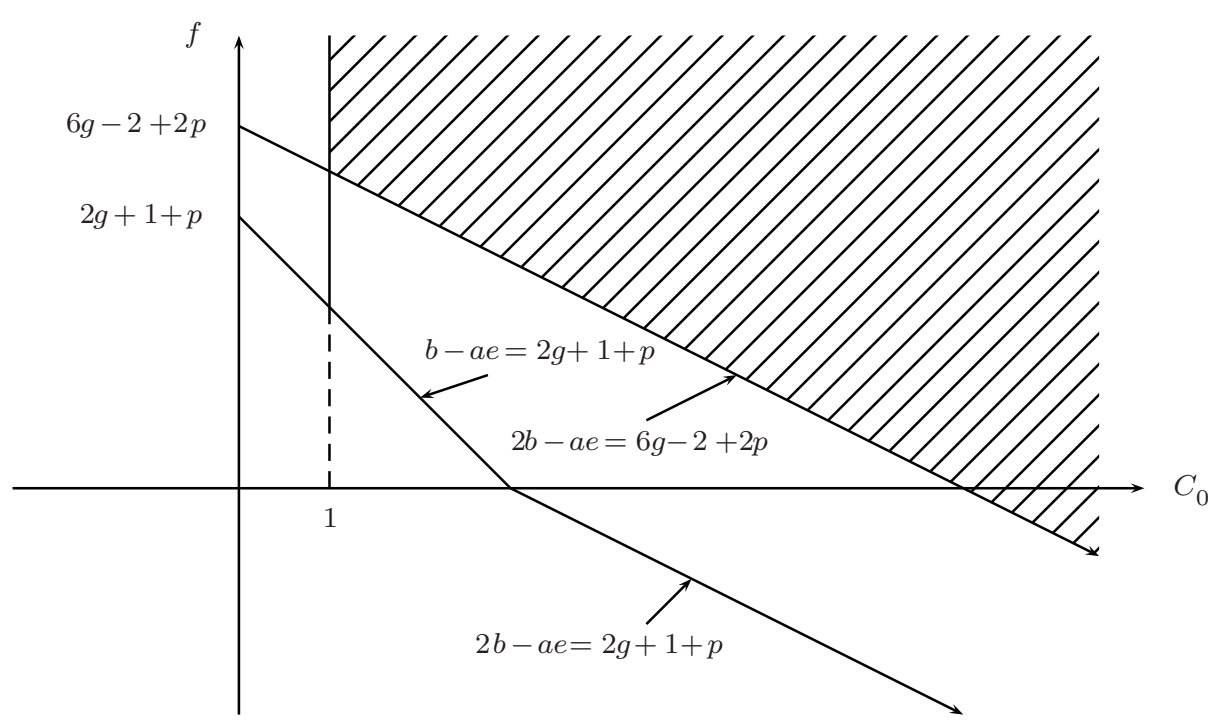

FiguRE 3. Ruled surface with $e<0$

\section{REFERENCES}

[1] David C. Butler, Normal generation of vector bundles over a curve, J. Differ. Geom. 39 (1994), 1-34. MR.1258911 (94k:14024)

[2] David Eisenbud, The Geometry of Syzygies, Graduate Texts in Math., no. 229, SpringerVerlag, New York (2005). MR2103875 (2005h:13021)

[3] F. J. Gallego and B. P. Purnaprajna, Normal Presentation on Elliptic Ruled Surfaces, J. Algebra 186 (1996), 597-625. MR1423277 (98c:14030) 
[4] F. J. Gallego and B. P. Purnaprajna, Higher syzygies of elliptic ruled surfaces, J. Algebra 186 (1996), 626-659. MR 1423278 (98c:14031)

[5] F. J. Gallego and B. P. Purnaprajna, Projective normality and syzygies of algebraic surfaces, J. Reine Angew. Math. 506 (1999), 145-180. MR1665689 (2000a:13023)

[6] F. J. Gallego and B. P. Purnaprajna, Vanishing theorems and syzygies for K3 surfaces and Fano varieties, J. Pure App. Alg. 146 (2000), 251-265. MR1742342 (2001f:14071)

[7] F. J. Gallego and B. P. Purnaprajna, Some results on rational surfaces and Fano varieties, J. Reine Angew. Math. 538 (2001), 25-55. MR.1855753 (2002f:14024)

[8] M. Green, Koszul cohomology and the geometry of projective varieties I, J. Differ. Geom. 19 (1984), 125-171. MR0739785 (85e:14022)

[9] M. Green and R. Lazarsfeld, On the projective normality of complete linear series on an algebraic curve, Inv. Math. 83 (1986), 73-90. MR0813583 (87g:14022)

[10] M. Green and R. Lazarsfeld, Some results on the syzygies of finite sets and algebraic curves, Compositio Mathematica 67 (1988), 301-314. MR0959214 (90d:14034)

[11] Robin Hartshorne, Algebraic Geometry, no. 52, Springer-Verlag, New York (1977). MR0463157 (57:3116)

[12] Y. Homma, Projective normality and the defining equations of ample invertible sheaves on elliptic ruled surfaces with $e \geq 0$, Natural Sci. Rep. Ochanomizu Univ. 31 (1980), 61-73. MR.0610593 (82e:14044)

[13] Y. Homma, Projective normality and the defining equations of an elliptic ruled surface with negative invariant, Natural Sci. Rep. Ochanomizu Univ. 33 (1982), 17-26. MR0703959 (85a:14027)

[14] Euisung Park, On higher syzygies of ruled varieties over a curve, to appear in J. Algebra.

School of Mathematics, Korea Institute for Advanced Study, 207-43 Cheongryangri 2-Dong, Dongdaemun-gu, Seoul 130-722, Republic of Korea

E-mail address: puserdos@kias.re.kr 